\title{
Methodenvorstellung
}

\section{Gruppenpuzzle - Didaktische Gestaltung und Illustration aus der Lehrerbildung}

\author{
Karl-Heinz Gerholz*
}

\section{A. Angaben zur Veranstaltung}

- Hochschule: Universität Paderborn

- Veranstaltungsart: Modul mit Input-, Gruppen- und Selbststudiumsphasen

- Größe: 120 Studierende, 5. Fachsemester, 5 ECTS (150 h)

- Räumlichkeit: Vorlesungssaal sowie kleinere Räume für Expertengruppen

- Materialien: abhängig vom Einsatz der Methode (u.a. Texte)

- Zeitaufwand: abhängig von didaktischer Gestaltung (z.B. Vorbereitung 2 h, Durchführung in einer Modulsitzung)

\section{B. Beschreibung der Methode}

Das Gruppenpuzzle kann den Methoden des kooperativen und problembasierten Lernens zugeordnet werden. Die Grundidee ist dabei, dass eine Problemstellung kooperativ in drei Arbeitsphasen bearbeitet wird. In den Arbeitsphasen wechselt die Gruppenzusammensetzung in Stamm- und Expertengruppen, womit ein Wechselspiel zwischen Problembearbeitung und Aneignung von Expertise modelliert wird. Die Methodik wurde in den 1970er Jahren von Aronson et al. (1978) als ,Jigsawtechnique' entwickelt, indem ein Rahmenthema in unterschiedliche Bereiche ,zersägt' (engl. saw) und dann wieder als Puzzle (engl. jigsaw) zusammengesetzt wird. ${ }^{1}$ Die Abbildung visualisiert den Arbeitsprozess in den Gruppen.

* Der Autor ist Juniorprofessor für Wirtschaftspädagogik, insbesondere Hochschuldidaktik und -entwicklung an der Universität Paderborn.

1 Aronson/Blaney et al., The jigsaw classroom, Beverly Hills 1978. 

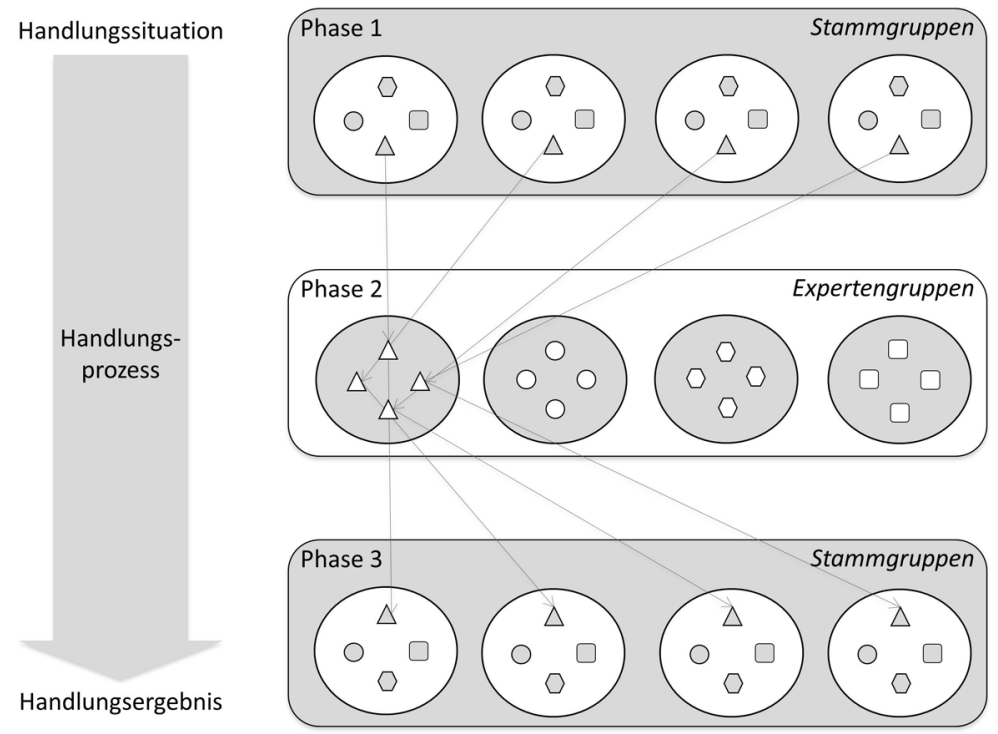

In Phase 1 werden die Stammgruppen mit einer Problemstellung (z.B. Fallstudie) konfrontiert. Zur Bearbeitung werden unterschiedliche fachliche Kenntnisse benötigt, wofür jeweils ein oder mehrere Experten bestimmt werden.

In Phase 2 entsenden die Stammgruppen ihre Mitglieder in die unterschiedlichen Expertengruppen. Es geht um die Erkundung und Erarbeitung der einzelnen fachlichen Themen. Dies kann auf Basis von Texten, Inputsequenzen u. Ä. organisiert werden. Ziel ist es, dass sich die Expertengruppen einen spezifischen Themenbereich aneignen. Die Studierenden sollen in dieser Phase zu ,Experten’ werden.

In Phase 3 finden sich die Stammgruppen wieder mit ihren nun jeweiligen Experten zusammen. In dieser Phase geht es auf Basis der nun vorhandenen Expertise um die Entwicklung von Handlungsmöglichkeiten für die Problemstellung und die begründete Entscheidung für eine Lösung. Letzteres stellt das Handlungsergebnis der Gruppe dar, welches präsentiert und reflektiert wird.

Aus lerntheoretischer Perspektive wird im Gruppenpuzzle Lernen als situationsgebundener, aktiv konstruierender und sozialer Prozess modelliert. Die Studierenden werden mit einer Problemsituation (Handlungssituation) konfrontiert, die sie kooperativ über die Stamm- und Expertengruppen bearbeiten (Handlungsprozess). Anschließend werden die Ergebnisse im Plenum präsentiert (Handlungsergebnis). ${ }^{2}$ 


\section{Ziele der Methode}

Die Methode des Gruppenpuzzles als kooperative Lernform kann unterschiedliche Lernziele in didaktischen Arrangements unterstützen. Aus fachwissenschaftlicher Perspektive kann die soziale Interaktion eine vertiefende Auseinandersetzung mit dem Lerngegenstand fördern. Durch das Element der Expertengruppen werden die Studierenden aufgefordert, sich nicht nur neues Wissen anzueignen, sondern dieses auch in ihren Stammgruppen zu explizieren, was Elemente der Zusammenfassung, Elaboration, Erläuterung und Einordnung von Wissen umfasst. ${ }^{3}$ Durch die unterschiedlichen Experten in den Stammgruppen werden die Studierenden angeregt, einen Sachverhalt - die Problemsituation - aus unterschiedlichen Perspektiven zu analysieren und zu bearbeiten.

Aus sozial-kommunikativer Perspektive wird beim Gruppenpuzzle die Gestaltung sozialer Interaktionsprozesse unterstützt. Die Stammgruppen erarbeiten sich gemeinsam eine Wissensbasis, in deren Prozess sie auf die einzelnen Gruppenmitglieder - die als Experten fungieren - angewiesen sind, weshalb die Problembearbeitung nur auf Basis einer kooperativen Arbeitsstruktur gelingen kann. Vor allem die Vielfalt der Beziehungen - hier in den Stamm- und Expertengruppen - kann zu einer verstärkten Förderung der sozial-kommunikativen Fähigkeiten beitragen.

\section{Beispiel zur Umsetzung: Selbstreguliertes Lernen in der Lehrerbildung}

Die Methode des Gruppenpuzzles wurde im Rahmen des Moduls ,Kompetenzentwicklung' (5. Fachsemester) in einem Lehramtsstudiengang durchgeführt. Das Thema stellte selbstreguliertes Lernen dar und im Mittelpunkt stand die Förderung von Lern- und Arbeitsstrategien. Unter selbstreguliertem Lernen wird die intentionale Verantwortungsübernahme für den eigenen Lernprozess verstanden, was mit der Beherrschung kognitiver (u.a. Informationsverarbeitungsstrategien), metakognitiver (u.a. Kontrolle des eigenen Lernprozesses) und motivationaler Strategien (z.B. sich motivieren können) einhergeht.

Phase 1 (1. Modulsitzung, 4h): ${ }^{4}$ Die Stammgruppen wurden mit der Problemsituation konfrontiert, eine Lernzeitschrift für Erstsemester zu konzipieren, mit deren Hilfe sich die Erstsemester Lerntechniken für das Studium aneignen. Es ging um die Förderung des selbstregulierten Lernens zu Beginn des Studiums. Anschließend wurde über einen Inputvortrag eine Einführung in das selbstregulierte Lernen gegeben.

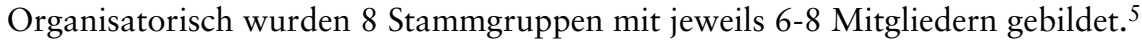

Phase 2 (2. Modulsitzung und Selbstlernphasen, ca. 8h): Innerhalb der Expertengruppen eigneten sich die Studierenden das notwendige Wissen zu Lernstrategien

3 Vgl. Renkl, in Wilk/Möller (Hrsg.), Heidelberg 2009, S. 3; Tan/Gallo et al., in: The Internet TESL Journal 1999, S. 1.

4 Nachfolgende Stundenangaben (h) beziehen sich jeweils auf Präsenz- und Selbststudiumszeiten.

5 Die Situation ist optimal, wenn die Mitgliederzahl der Stammgruppe gleich zur Anzahl der Expertengruppen ist, was aber in Modulen nicht immer aufgeht, weshalb es auch möglich ist, dass in einer Stammgruppe mehrere Mitglieder in dieselbe Expertengruppe entsendet werden. 
und deren Förderung an. Zum Beispiel beschäftige sich eine Expertengruppe mit dem Thema Informationsverarbeitungsstrategien, wozu u.a. Wiederholungsstrategien (z.B. mehrmaliges Lesen) und Elaborationsstrategien (z.B. Bildung von Assoziationen) gehören. Die anderen Expertengruppen befassten sich u.a. mit Themen der ,Förderung von selbstreguliertem Lernen' oder ,Regulationsstrategien' zur Steuerung des Lernprozesses. Hierzu wurden den Expertengruppen ausgewählte Texte zur Verfügung gestellt, die sie bearbeiteten. Insgesamt gab es 8 Expertengruppen, die über unterschiedliche Seminarräume verteilt wurden.

Phase 3 (3. Modulsitzung und Selbstlernphasen, ca. 10h): Zurück in den Stammgruppen mussten die Studierenden sich gegenseitig ihr angeeignetes Wissen weitergegeben und dieses problemorientiert im Sinne der Gestaltung einer Lernzeitschrift für Erstsemester anwenden. Die Lernzeitschriften wurden anschließend im Plenum präsentiert und hinsichtlich der didaktischen Ausgestaltung diskutiert.

\section{E. Potentiale und Herausforderungen des Gruppenpuzzles}

Das Gruppenpuzzle stellt eine Methode dar, die sowohl innerhalb einer Modulsitzung als auch über eine Sequenz von Modulsitzungen zum Einsatz gelangen kann. Die konkrete Gestaltung ist in Abhängigkeit der didaktischen Entscheidungsfelder vorzunehmen: Welche Kompetenzen sollen gefördert werden (z.B. kommunikative Fähigkeiten, Aufbau eines vertieften Fachwissens)? Welche Inhalte sollen sich die Studierenden aneignen (z.B. Lerntechniken, Lernform ,selbstreguliertes Lernen')? Wie wird der Lernprozess durch Lehrinterventionen begleitet (z.B. Inputvorträge, Coaching)? Welche Medien unterstützen den Lernprozess (z.B. Fachtexte, Videos)?

So wäre es im Bereich der Rechtswissenschaften denkbar, die Studierenden mit einer Fallsituation zum Erbrecht zu konfrontieren (z.B. Vererbung eines Hauses in Spanien an die Söhne in Deutschland). Innerhalb der Expertengruppen können nun Unterthemen wie ,Bereich Erbrecht im BGB', ,Rechtsprechung am BGH', ,Münchener Kommentar' oder ,EU-Erbrechtsverordnung' erkundet werden. Die Expertengruppen können sich das Wissen z.B. über Texte, Aufforderung zur eigenen Recherche im Internet oder Inputvorträge des Lehrenden aneignen. Ziel ist es, dass innerhalb der Stammgruppen eine Lösung zur Fallsituation erarbeitet wird, die mit einer Präsentation (z.B. Posterpräsentation) und/oder Prüfungsleistung (z.B. Hausarbeit, Reflexionsbericht) beendet wird.

Die Methode des Gruppenpuzzles bietet unterschiedliche Integrationsvarianten in einem Modul. Hinsichtlich der verfolgten Lernziele können die Förderung von Textverständnis, die Stärkung von sozial-kommunikativen Fähigkeiten oder die Wissensvertiefung und -anwendung im Vordergrund stehen. Die methodische Integration kann von dem Einsatz in einer Modulsitzung über die Gestaltung der Expertengruppenphase über Selbststudiumsphasen bis zum Einsatz über eine gesamte Modulsequenz variieren. Bei der erstmaligen Erprobung der Methode wäre es denkbar, zu- 
nächst ein kleines Arrangement zu wählen, um darüber Erfahrungen für den zukünftigen Einsatz des Gruppenpuzzles zu gewinnen.

\section{Weiterführende Literaturhinweise}

Aronson, Elliot et al., The jigsaw classroom. Beverly Hills, CA: Sage 1978.

Ghaith, Ghazi/El-Malak, Mirna Abd, Effect of Jigsaw II on Literal and Higher Order EFL Reading Comprehension, in: Educational Research and Evaluation: An International Journal on Theory and Practice 2004, S. 105-115.

Online: www.jigsaw.org 